\title{
1245+676 - A CSO/GPS Source being an Extreme Case of a Double-Double Structure
}

\author{
Andrzej Marecki ${ }^{1}$, Peter D. Barthel ${ }^{2}$, Antonis Polatidis ${ }^{3}$ and Izabela Owsianik ${ }^{3, *}$ \\ ${ }^{1}$ Toruń Centre for Astronomy, Nicholas Copernicus University, Toruń, Poland \\ ${ }^{2}$ Kapteyn Institute, Department of Astronomy, Groningen University, The Netherlands \\ ${ }^{3}$ Max-Planck-Institut für Radioastronomie, Bonn, Germany
}

Received 2002 July 2, accepted 2002 September 16

\begin{abstract}
AGN with the so-called 'double-double' radio structure have been interpreted as restarted AGN where the inner structure is a manifestation of a new phase of activity which happened to begin before the outer radio lobes resulting from the previous one had faded completely. The radio galaxy $1245+676$ is an extreme example of such a double-double object - its outer structure, measuring $970 \mathrm{~h}^{-1} \mathrm{kpc}$, is five orders of magnitude larger than the $9.6 h^{-1} \mathrm{pc}$ inner one. We present a series of VLBI observations of the core of $1245+676$ which appears to be a compact symmetric object (CSO). We have detected the motion of the CSOs lobes, measured its velocity, and inferred the kinematic age of that structure.
\end{abstract}

Keywords: galaxies: active — galaxies: individual (1245+676)

\section{Objects with the 'Double-Double' Radio Structure}

Radio galaxies which are not beamed toward the observer are normally perceived as double structures. The majority of them are large scale objects (LSOs) with angular sizes ranging from several arcseconds to several arcminutes. These angular sizes translate to large linear sizes of the order of $10^{5}-10^{6} \mathrm{pc}$; galaxies with sizes $>1 \mathrm{Mpc}$ are labelled 'giant radio galaxies' (GRGs). A very interesting exception to this (relatively simple) picture exists however: a few LSOs, which are also double at first sight, turn out to have so-called double-double structure, radio galaxy $1450+333$ (Schoenmakers et al. 2000) being a prime example.

LSO radio lobes are powered by central engines for a maximum of approximately $10^{7} \mathrm{yr}$ (Alexander \& Leahy 1987; Liu, Pooley, \& Riley 1992). If the nuclear energy supply stops, the extended radio structure will stop growing, its luminosity drops and the spectrum gradually gets steeper and steeper because of radiation and expansion losses. Komissarov \& Gubanov (1994) estimate fadeaway time scales of several $10^{7} \mathrm{yr}$ which is comparable to the timescale of the activity itself.

After that time the radio structure should in principle disappear, however there are a number of known mechanisms of restarting activity. For example, Hatziminaoglou, Siemiginowska, \& Elvis (2001) elaborated a theory of thermal-viscous instabilities in the accretion disks of supermassive black holes (SMBHs). It predicts that the activity is recurrent and the length of the activity phase as well as the timescale of the activity re-occurrence is controlled by the mass of the SMBH. In favourable circumstances a new phase of activity can start before

\footnotetext{
* Present address: Institute of Geodesy, University of Bonn, Germany.
}

the radio lobes resulting from the previous one have faded completely. This would lead to double-double radio structure.

\section{Inner and Outer Radio Structures of $1245+676$}

$1245+676$ is a classical double-lobed radio galaxy redshifted to $z=0.1073$. Its angular size amounts to 12 '.4 (Lara et al. 2001) which is equivalent to $970 h^{-1} \mathrm{kpc}$ (assuming $q_{0}=0.5$ ). Figure 1 shows the NVSS map of $1245+676$. The central component is noticeably strong. Indeed, according to Lara et al. (2001) 2/3 of the whole flux at $1.4 \mathrm{GHz}$ is emitted by the core. Moreover the core

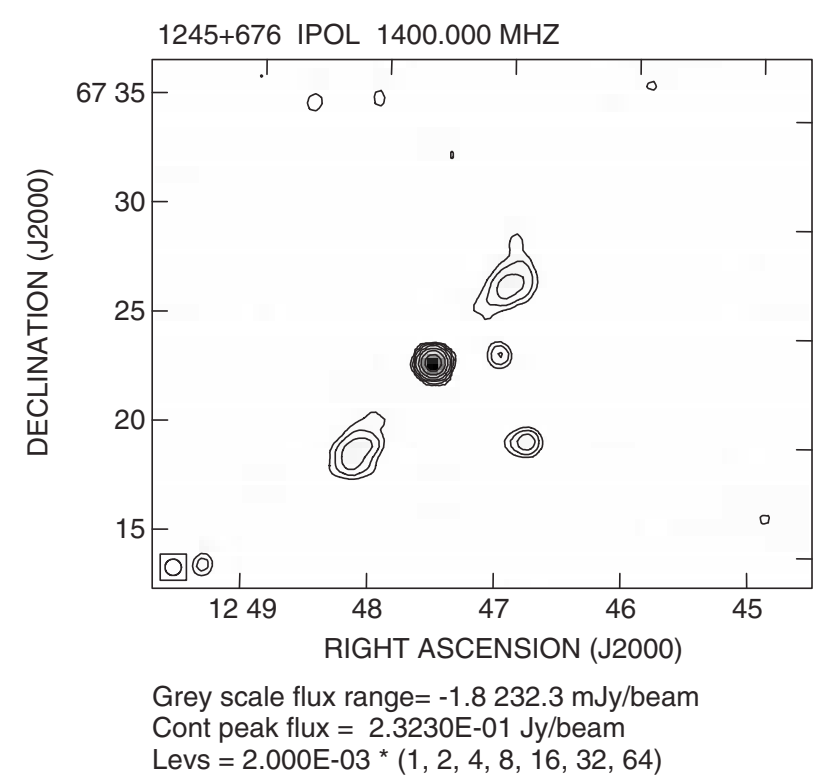

Figure 1 NVSS image of the whole structure of the radio galaxy $1245+676$. The radio galaxy stretches SE-NW over 12 ' 4 - also two compact field sources are seen. 
has a gigahertz-peaked spectrum (GPS) - see for example the spectrum shown in Marecki et al. (1999).

A number of VLBI observations of the core of $1245+676$ have been conducted in different epochs. More technical details about these observations will be given elsewhere. In Figure 2 we present a selection of resulting $5 \mathrm{GHz}$ images; in Figure 3 we show the $8.4 \mathrm{GHz}$ map made in 1991 and the most detailed VLBI map of the 1245+676 core observed in 1998 at $15 \mathrm{GHz}$. These maps clearly show that the 'core' is actually a compact symmetric object
(CSO) with a double-lobed inner structure. Its size is only $9.6 h^{-1}$ pc. This means $1245+676$ is an extreme case of a double-double object - its outer structure is five orders of magnitude larger than the inner one.

We are confident that the core of $1245+676$ is indeed a CSO because based on our dual frequency ( 5 and $15 \mathrm{GHz}$ ) quasi-simultaneous VLBA observations made on 6 September 1998 we were able to calculate the spectral indices of the milliarcsecond scale components. They amount to $\alpha_{\text {North }}=-1.03$ and $\alpha_{\text {South }}=-1.23$ so they are

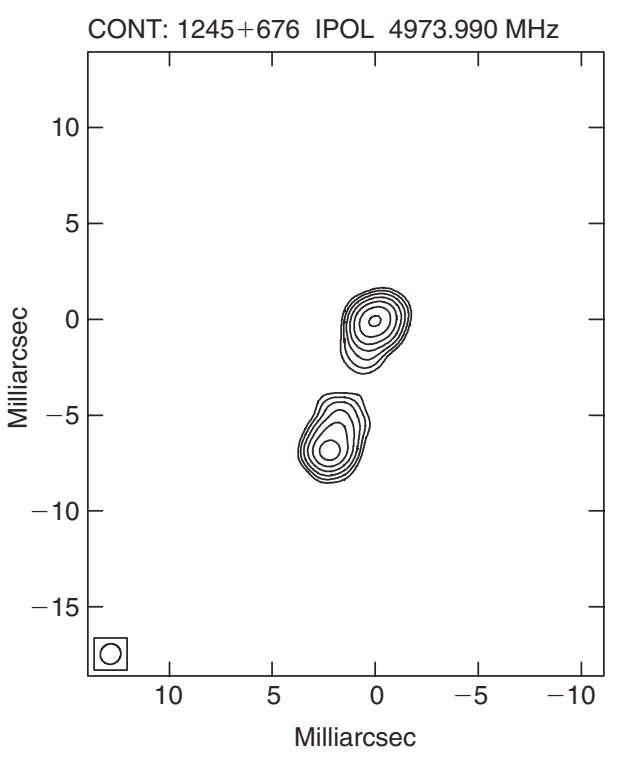

Center at RA 124733.32787 DEC 672316.4587 Cont peak flux $=3.6090 \mathrm{E}-02 \mathrm{Jy} / \mathrm{beam}$

Levs $=5.000 \mathrm{E}-04^{*}(-1,1,2,4,8,16,32,64,128,256)$

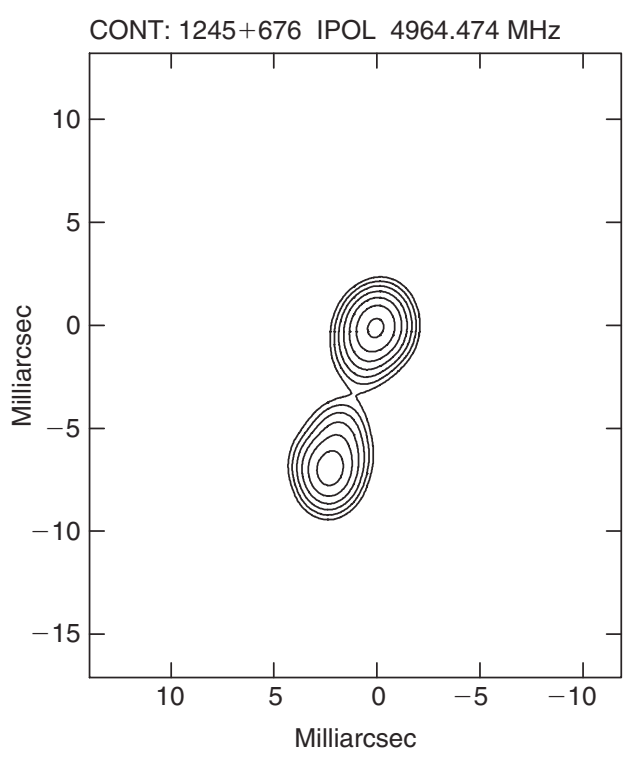

Center at RA 124733.32819 DEC 672316.4588 Cont peak flux $=7.4095 \mathrm{E}-02 \mathrm{Jy} / \mathrm{beam}$ Levs $=1.000 \mathrm{E}-03^{*}(-1,1,2,4,8,16,32,64,128,256)$

Figure 2 VLBI images of $1245+676$ at $5 \mathrm{GHz}$ for epochs 1991 and 1998.

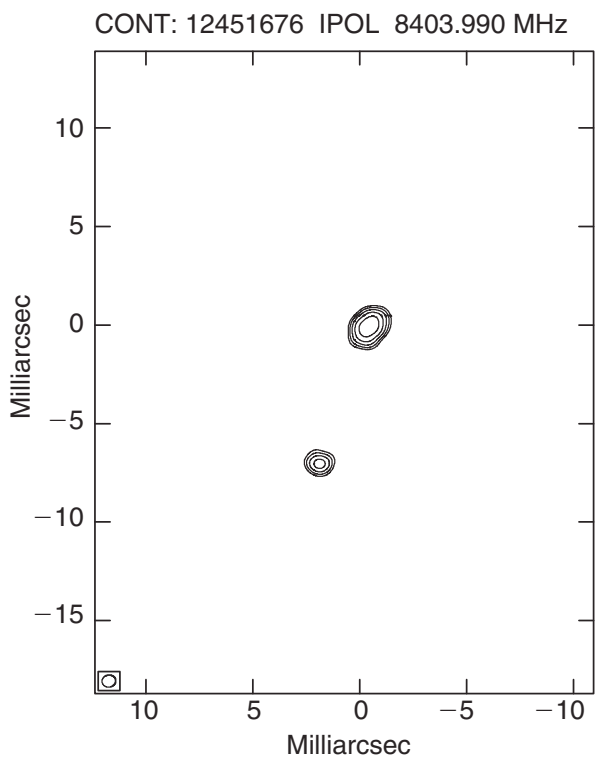

Center at RA 124733.32778 DEC 672316.4588 Cont peak flux $=2.6896 \mathrm{E}-02 \mathrm{Jy} / \mathrm{beam}$

Levs $=1.000 \mathrm{E}-03^{*}(-1,1,2,4,8,16,32,64,128,256)$

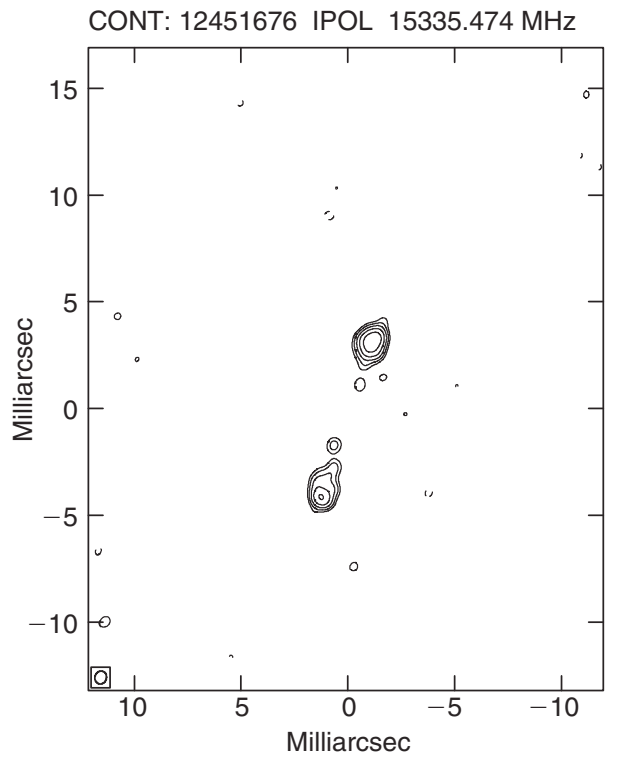

Center at RA 124733.32839 DEC 672316.4548 Cont peak flux $=1.2134 \mathrm{E}-02 \mathrm{Jy} / \mathrm{beam}$

Levs $=4.000 \mathrm{E}-04^{*}(-1,1,2,4,8,16,32,64,128,256)$

Figure 3 VLBI images of $1245+676$ at $8.4 \mathrm{GHz}$ (epoch 1991) and $15 \mathrm{GHz}$ (epoch 1998). 
both steep and roughly equal. We can therefore safely rule out a possibility they are not lobes but, for example, a core and a jet. (This approach is similar to that adopted by Peck \& Taylor 2000.)

We note a modest misalignment between the parsec scale $\left(P A=-19^{\circ}\right)$ and the megaparsec scale $\left(P A=-50^{\circ}\right)$ which may be attributed to precession of SMBH and the accretion disk.

\section{Expansion Velocity and the Kinematic Age}

Given the substantial time baseline between the $5 \mathrm{GHz}$ observations we attempted the measurement of the lobes' expansion velocity. We used different software tools from AIPS and Difmap packages, and the final expansion figure is an average of the measurements obtained. It amounts to

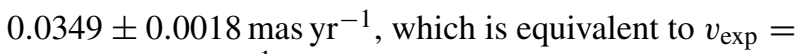
$0.164 \pm 0.008 h^{-1} \mathrm{c}$. The inferred kinematic age of the CSO structure is $191 \mathrm{yr}$.

\section{References}

Alexander, P., \& Leahy, J. P. 1987, MNRAS, 225, 1

Hatziminaoglou, E., Siemiginowska, A., \& Elvis, M. 2001, ApJ, 547, 90

Komissarov, S. S., \& Gubanov, A. G. 1994, A\&A, 285, 27

Lara, L., Cotton, W. D., Feretti, L., Giovannini, G., Marcaide, J. M., Márquez, I., \& Venturi, T. 2001, A\&A, 370, 409

Liu, R., Pooley, G. G., \& Riley, J. M. 1992, MNRAS, 257, 545

Marecki, A., Falcke, H., Niezgoda, J., Garrington, S. T., \& Patnaik, A. R. 1999, A\&AS, 135, 273

Peck, A. B., \& Taylor, G. B. 2000, ApJ, 534, 90

Schoenmakers, A. P., de Bruyn, A. G., Röttgering, H. J. A., van der Laan, H., \& Kaiser, C. R. 2000, MNRAS, 315, 371 